\title{
Bem-estar animal no manejo pré-abate e a influência na qualidade da carne suína e nos parâmetros fisilógicos do estresse
}

\author{
Animal welfare at pre-slaughter handling and the influence on pork meat quality and on stress \\ physiological parameters
}

\author{
Charli Beatriz Ludtke ${ }^{\mathrm{I}}$ Osmar Antonio Dalla CostaII Roberto de Oliveira Roça'II \\ Expedito Tadeu Facco Silveira ${ }^{\mathrm{IV}}$ Natália Bortoleto Athayde ${ }^{\mathrm{I}}$ Aurélia Pereira de Araújo $^{\mathrm{I}}$ \\ Amilton de Mello Júnior ${ }^{I}$ Natália Chinellato de Azambuja ${ }^{I V}$
}

\section{RESUMO}

Avaliaram-se os efeitos do sistema de embarque/ desembarque (S1: veículo 1, carroceria com piso móvel e uso de prancha de manejo para condução dos suínos; S2: veículo 2, carroceria com piso fixo e uso de bastão elétrico para condução dos suínos) e da genética ( $A, B$ e $C$ ) sobre as características físico-químicas da carne, lesões na pele $e$ parâmetros fisiológicos do estresse. Foram utilizados 120 suínos machos castrados (peso médio de $115 \mathrm{~kg}$ ) provenientes de três granjas divididos em seis grupos $(n=20)$ e submetidos a dois tipos de veículos para o transporte. Para a análise estatística, consideram-se, no modelo de análise da variância, os efeitos de blocos (dias de avaliação), sistema de embarque/ desembarque, genética $e$ interação entre as linhagens genéticas e modelos dos veículos dispostos no esquema fatorial (2 modelos de veículos $x 3$ linhagens genéticas). Os valores médios

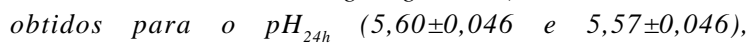
cor $^{*}{ }_{24 h}(41,52 \pm 1,256$ e 41,21 $\pm 1,486)$, perda por exsudação

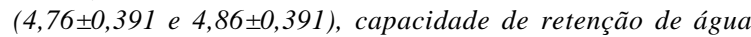
$(0,038 \pm 0,03$ e 0,039 $\pm 0,003)$ não demonstraram diferença entre os sistemas de embarque e desembarque (S1 e S2) nas genéticas $A, B$ e $C$. A incidência de escoriações de pele no pernil, corpo e paleta não diferiram entre os métodos de embarque e desembarque nas genéticas $A, B$ e $C$. Ambos os sistemas $S 1$ e $S 2$ geraram baixos níveis de estresse $e$ proporcionaram facilidade de manejo nas genéticas avaliadas.

Palavras-chave: bem-estar animal, embarque, qualidade de carne, suínos, transporte.

\begin{abstract}
The effects of the loading/unloading systems have been evaluated (S1: vehicle 1, with mobile floor and using management board for conducting pigs, S2: vehicle 2, with fixed floor and using electric goads to conduct pigs) and genetic ( $A, B$ and $C$ ) on the physiochemical characteristics of the meat, skin damage and physiological parameters of stress. 120 castrated pigs have been used (average weight $115 \mathrm{~kg}$ ) from three farms, divided into six groups $(n=20)$ and subjected to two types of transport vehicles. For statistical analysis the effects of blocks (assessment days) have been considered in the variance analysis model), loading/unloading systems, genetics and the engagement among the genetic lineages and vehicle models arranged in a factorial scheme (2 vehicle models $x 3$ genetic lineages). The average values obtained for the $\mathrm{pH}_{24 \mathrm{~h}}$

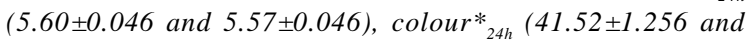
$41.21 \pm 1.486)$, drip loss (4.76 \pm 0.391 and $4.86 \pm 0.391)$, water holding capacity $(0.038 \pm 0.03$ and $0.039 \pm 0.003)$, have not shown differences between the loading and unloading systems (S1 and S2) in the genetic lineages A, B and C. The skin damage incidence on the leg, middle and shoulder haven't differed between the loading and unloading systems in the genetic lineages A, B and C. Both S1 and S2 systems have shown low stress levels and provided easiness at handling among the assessed lineages.
\end{abstract}

Key words: animal welfare, loading, meat quality, pigs, transport.

IPós-graduação em Medicina Veterinária e Zootecnia, Universidade Estadual Paulista Julio de Mesquita Filho (UNESP), Botucatu, SP, Brasil.

IIEmpresa Brasileira de Pesquisa Agropecuária, Embrapa Suínos e Aves (CNPSA), BR153, Km 110, CP 21, Distrito de Tamanduá, 89700-000, Concórdia, SC, Brasil. E-mail: osmar@cnpsa.embrapa.br. Autor para correspondência.

IIIFaculdade de Ciências Agronômicas, UNESP, Botucatu, SP, Brasil.

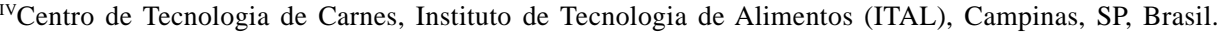




\section{INTRODUÇÃO}

O transporte dos animais para o abate tem recebido considerável atenção pelas indústrias e órgãos governamentais nos últimos anos. A Comissão da União Europeia publicou o regulamento de proteção dos animais durante o transporte que estabelece normas específicas para atender o bem-estar destes (EC, 2005). A Organização Mundial de Saúde Animal (OIE, 2011) também adotou novos padrões para a proteção dos animais durante o transporte. Desse modo, há a necessidade da revisão e atualização das regulamentações para o transporte dos animais com perspectivas de melhorias no bem-estar e manutenção no mercado internacional (BENCH et al., 2008). No entanto, as condições de transporte em cada país são extremamente variáveis, diferindo no manejo antes, durante e após o transporte.

O transporte é uma situação estressante para os suínos, pois expõe os animais a novos fatores potencialmente estressantes, como dificuldades no embarque e desembarque, barulhos, vibrações, mudanças de velocidade brusca do caminhão e variações na temperatura ambiental. Esses fatores de estresse, frequentemente, levam às respostas comportamentais e fisiológicas que podem contribuir para a redução de rendimento da carcaça e qualidade da carne (BENCH et al., 2008).

O bem-estar dos suínos durante o transporte pode ser influenciado pelo modelo do veículo. Diversos países no Reino Unido (Inglaterra) substituíram os veículos com carrocerias convencionais por veículos com carrocerias com piso móvel para facilitar o embarque e desembarque dos suínos, reduzir a ocorrência de intervenções agressivas e permitir a substituição do bastão elétrico pela prancha de manejo.

O estresse é o principal parâmetro utilizado para avaliar o bem-estar animal. A maioria dos autores, entre os quais se destaca GRANDIN (1998), descreve que, sob estresse, os animais desenvolvem mecanismos de respostas, quando sua homeostasia está ameaçada, necessitando de ajustes fisiológicos ou comportamentais para adequar-se aos aspectos adversos do manejo ou ambiente. Há pelo menos dois sistemas para medir o estresse. Um deles é através do comportamento e o outro pela avaliação dos parâmetros biológicos (respostas endócrinas e enzimáticas) nos fluídos ou músculos dos animais. No caso dos animais para o abate, as informações do estresse ante-mortem, podem ser avaliadas na carcaça (SHAW \& TUME, 1992).

Este trabalho teve como objetivo avaliar o efeito do sistema de embarque/desembarque sobre a incidência de lesões nas carcaças, qualidade da carne e parâmetros fisiológicos do estresse em três linhagens genéticas de suínos.

\section{MATERIAL E MÉTODOS}

O experimento foi realizado durante o verão na região oeste de Santa Catarina, próximo à cidade de Joaçaba, com temperatura variando entre 22 a $31^{\circ} \mathrm{C}$ e a umidade relativa do ar de 74 a $78 \%$. Foram utilizados 120 suínos machos, castrados, pesando em média 115kg e provenientes de cruzamentos industriais de três linhagens genéticas A, B e C (comercializadas no Brasil), com predomínio das cruzas entre Large White e Landrace. Esses animais foram divididos em seis grupos $(n=20)$ e submetidos a dois sistemas de embarque e desembarque para transporte ao frigorífico.

Os animais foram criados em três diferentes granjas no sistema de terminação, com distância do frigorífico variando de 100 a $120 \mathrm{~km}$. O transporte dos suínos foi realizado em dois modelos de veículos com carrocerias duplas metálicas com piso móvel (S1) ou piso fixo (S2), desenvolvidas pela empresa TRIEL-HT, ambas com capacidade para transportar 96 suínos em 16 compartimentos. Para realizar o embarque e desembarque dos suínos no $2^{\circ}$ piso da carroceria com piso fixo, foi colocada uma rampa de metal (3m de comprimento). Na carroceria com piso móvel não foi utilizada rampa de metal, somente a rampa de acesso às instalações da granja, já que o $2^{2}$ piso se adéqua à altura do $1^{\underline{0}}$ piso. O veículo 1 possuía um sistema de embarque e desembarque, com carroceria com piso móvel (S1) e o veículo 2 apresentava o embarque e desembarque convencional, com carroceria com piso fixo (S2). Durante os procedimentos de embarque e desembarque no S1, utilizou-se a prancha de manejo para condução e para o S2 foi utilizado bastão elétrico. Todos os animais foram submetidos ao jejum de 12 horas pré-embarque, transportados à noite e permaneceram sob dieta hídrica durante o tempo de descanso (6h). Para a insensibilização dos suínos, utilizaram-se eletrodos na região das têmporas, com descarga elétrica de 700 volts durante 3 segundos e corrente elétrica de 1,3ampér. Na sequência, os animais foram abatidos através de incisão da veia jugular e artéria carótida e sangrados (mesa rolante). Separaramse aleatoriamente 120 suínos nas baias de descanso, incluindo ambos os grupos ( $\mathrm{n}=20$ suínos por grupo), para avaliação de parâmetros de qualidade da carcaça (avaliações físico-químicas e visual). Todas as avaliações nas carcaças foram realizadas no período de 24 h post-mortem. 
Os índices de lesões de pele foram obtidos visualmente no pernil, paleta e corpo das carcaças, baseando-se em um guia padrão de fotos (1-ausência, 2-leve; 3-moderada e 4-severa), conforme descrito por BARTON-GADE et al. (1996). As mensurações de $\mathrm{pH}$ (pHmêtro - INGOLD-WTW) e de cor (colorímetro Minolta- DL65, modelo CR 300), no sistema $\mathrm{L}^{*}$ (luminosidade) $\mathrm{a}^{*}$ (intensidade da cor vermelha) $\mathrm{b}^{*}$ (intensidade da cor amarela), foram realizadas em duplicata no músculo Semimembranosus, no período de 2 e 24h post-mortem. Para as avaliações de perda por exsudação e capacidade retenção de água (CRA), foram utilizadas amostras em duplicatas do músculo Longissimus dorsi, entre a 2ª e a $4^{\mathrm{a}}$ vértebra torácica, num período de $24 \mathrm{~h}$ post-mortem. A avaliação da perda por exsudação foi baseada na suspensão da amostra em sacos plásticos inflados, sob atuação da gravidade, conforme metodologia descrita por HONIKEL (1998) e a capacidade de retenção de água foi realizada segundo a metodologia descrita por BERTOLONI et al. (2006b).

$\mathrm{Na}$ sangria, foram coletadas amostras de sangue para análises dos parâmetros fisiológicos do estresse (cortisol, creatina fosfoquinase e lactato). A avaliação do nível de cortisol do plasma foi realizada pela técnica de radioimunoensaio, com o kit TKCO1 / DPC-Medlab ), e as avaliações de creatina fosfoquinase no plasma foram realizadas com o kit CK-NAC / Laborlab e para lactato utilizou-se o kit lactat / Rolf
Greiner Biochemica. Ambas as avaliações foram realizadas conforme metodologia descrita por BERTOLONI et al. (2006a). Para as análises dos dados de qualidade de carne, lesões na pele e parâmetros fisiológicos do estresse, foram considerados como unidade experimental os modelos dos veículos de transporte. As médias obtidas foram avaliadas através da análise da variância e, para o modelo estatístico, foi utilizado o delineamento de blocos (dias de avaliação i = 1 e 2 ) completamente casualizados, com tratamentos dispostos no esquema fatorial (2 modelos de veículos x 3 linhagens genéticas), interação entre as linhagens genéticas e sistema de embarque e desembarque.

A análise foi realizada usando o procedimento GLM do Statistical Analysis System (SAS, 2003) e o detalhamento das análises para o efeito das linhagens genéticas foi realizado através do teste de Tukey (5\% de significância).

\section{RESULTADOS E DISCUSSÃO}

Os valores médios dos escores de lesões de pele na região do pernil, corpo e paleta demonstraram que as incidências foram de ausente a leve, não diferindo $(\mathrm{P}>0,05)$ para os sistemas de embarque e desembarque S1 e S2 (Tabela 1) e para as linhagens genéticas $\mathrm{A}$, B e C (Tabela 2).

Tabela 1 - Valores médios, erros padrão e níveis descritivos de probabilidade de lesões na pele, avaliações de qualidade de carne e parâmetros fisiológicos do estresse dos suínos submetidos aos tratamentos S1 e S2.

\begin{tabular}{|c|c|c|c|}
\hline Lesões na pele & $\mathrm{S} 1$ & $\mathrm{~S} 2$ & $\operatorname{Pr}>F$ \\
\hline Pernil & $0,531 \pm 0,090$ & $0,590 \pm 0,090$ & 0,6605 \\
\hline Corpo & $0,757 \pm 0,076$ & $0,896 \pm 0,076$ & 0,2511 \\
\hline Paleta & $0,612 \pm 0,050$ & $0,531 \pm 0,050$ & 0,3012 \\
\hline Media de lesões & $0,629 \pm 0,071$ & $0,672 \pm 0,071$ & 0,6835 \\
\hline $\mathrm{pH}(2 \mathrm{~h})-\mathrm{SM}$ & $\begin{array}{c}\text { Avallaçoes de q } \\
5,977 \pm 0,044\end{array}$ & $6,08 \pm 0,052$ & 0,1899 \\
\hline $\mathrm{pH}(24 \mathrm{~h})-\mathrm{SM}$ & $5,603 \pm 0,046$ & $5,578 \pm 0,046$ & 0,7195 \\
\hline Cor $\mathrm{L}^{*} \mathrm{SM}_{(2 \mathrm{~h})}$ & $41,522 \pm 1,256$ & $41,217 \pm 1,486$ & 0,883 \\
\hline Cor $\mathrm{a}^{*} \mathrm{SM}_{(2 \mathrm{~h})}$ & $10,290 \pm 0,748$ & $10,165 \pm 0,885$ & 0,9192 \\
\hline Cor b*SM $_{(2 \mathrm{~h})}$ & $-2,323 \pm 0,081$ & $-2,318 \pm 0,095$ & 0,9696 \\
\hline Cor L*SM $(24 \mathrm{~h})$ & $43,373 \pm 0,547$ & $43,427 \pm 0,547$ & 0,9472 \\
\hline Cor a*SM (24h) & $12,738 \pm 0,431$ & $13,522 \pm 0,431$ & 0,255 \\
\hline Cor b*SM & $-1,970 \pm 0,264$ & $-1,422 \pm 0,264$ & 0,2015 \\
\hline Perda por exsudação-LD (\%) & $4,761 \pm 0,391$ & $4,868 \pm 0,391$ & 0,8533 \\
\hline CRA- LD $\left(\mathrm{cm}^{2}\right)$ & $0,038 \pm 0,003$ & $0,039 \pm 0,003$ & 0,7873 \\
\hline $\mathrm{CPK}\left(\mathrm{U} \mathrm{L}^{-1}\right)$ & $1071,1 \pm 0,960$ & $\begin{array}{l}1375,1 \pm 0,960 \\
13\end{array}$ & 0,9430 \\
\hline Lactato (mg dl $\left.{ }^{-1}\right)$ & $96,079 \pm 5,543$ & $101,060 \pm 5,543$ & 0,5528 \\
\hline Cortisol $\left(\mathrm{mcg} \mathrm{dl}^{-1}\right)$ & $8,146 \pm 0,130^{\mathrm{a}}$ & $8,956 \pm 0,130^{\mathrm{b}}$ & 0,0007 \\
\hline
\end{tabular}

S1= sistema de embarque e desembarque utilizando veículo com carroceria com piso móvel e S2= sistema de embarque e desembarque utilizando veículo com carroceria com piso fixo. Lesões da pele são medidas por um escore de 4 pontos: 1 e 2 representam valores aceitáveis e 3 e 4 inaceitáveis. LD- Longissimus dorsi; SM- Semimembranosus; CRA= capacidade de retenção de água; CPK= creatina fosfoquinase.

Ciência Rural, v.42, n.3, mar, 2012. 
Tabela 2 - Valores médios, erros padrão e níveis descritivos de probabilidade das lesões na pele, qualidade de carne e parâmetros fisiológicos do estresse nas linhagens genéticas.

\begin{tabular}{|c|c|c|c|c|}
\hline Lesões na pele & Genética A & Genética B & Genética C & $\operatorname{Pr}>\mathrm{F}$ \\
\hline Pernil & $0,512 \pm 0,011$ & $0,512 \pm 0,111$ & $0,657 \pm 0,011$ & 0,596 \\
\hline Corpo & $0,760 \pm 0,093$ & $0,720 \pm 0,093$ & $0,999 \pm 0,093$ & 0,164 \\
\hline Paleta & $0,397 \pm 0,061$ & $0,453 \pm 0,061$ & $0,865 \pm 0,061$ & 0,056 \\
\hline \multirow[t]{2}{*}{ Media de lesões } & $0,557 \pm 0,087$ & $0,562 \pm 0,087$ & $0,833 \pm 0,087$ & 0,123 \\
\hline & \multicolumn{3}{|c|}{-------Avaliações de qualidade de carne--- } & - \\
\hline $\mathrm{pH}(2 \mathrm{~h})-\mathrm{SM}$ & $6,044 \pm 0,054$ & $5,984 \pm 0,068$ & $6,033 \pm 0,054$ & 0,677 \\
\hline $\mathrm{pH}(24 \mathrm{~h})-\mathrm{SM}$ & $5,549 \pm 0,056$ & $5,603 \pm 0,056$ & $5,618 \pm 0,056$ & 0,685 \\
\hline Cor L*SM $(2 \mathrm{~h})$ & $41,82 \pm 1,538$ & $41,631 \pm 1,945$ & $40,995 \pm 1,538$ & 0,961 \\
\hline Cor a*SM $(2 \mathrm{~h})$ & $10,229 \pm 0,916$ & $10,428 \pm 1,159$ & $10,027 \pm 0,916$ & 0,963 \\
\hline Cor b*SM $(2 \mathrm{~h})$ & $-2,203 \pm 0,099$ & $-2,027 \pm 0,125$ & $-2,732 \pm 0,099$ & 0,206 \\
\hline Cor L*SM $(24 \mathrm{~h})$ & $42,619 \pm 0,670$ & $44,235 \pm 0,670$ & $43,345 \pm 0,670$ & 0,317 \\
\hline Cor a*SM $(24 \mathrm{~h})$ & $13,021 \pm 0,528$ & $12,561 \pm 0,528$ & $13,808 \pm 0,528$ & 0,324 \\
\hline Cor b*SM ${ }_{(24 h)}$ & $-2,324 \pm 0,323$ & $-2,083 \pm 0,323$ & $-0,681 \pm 0,323$ & 0,310 \\
\hline Perda por exsudação- LD (\%) & $4,946 \pm 0,478$ & $4,834 \pm 0,478$ & $4,663 \pm 0,478$ & 0,917 \\
\hline CRA- LD $\left(\mathrm{cm}^{2}\right)$ & $0,041 \pm 0,004$ & $0,036 \pm 0,004$ & $0,039 \pm 0,004$ & 0,651 \\
\hline \multicolumn{5}{|c|}{ - } \\
\hline $\mathrm{CPK}\left(\mathrm{U} \mathrm{L}^{-1}\right)$ & $1375,75 \pm 9,66$ & $1488,93 \pm 9,66$ & $812,40 \pm 9,66$ & 0,845 \\
\hline Lactato (mg dl ${ }^{-1}$ ) & $92,429 \pm 6,788$ & $96,787 \pm 6,788$ & $106,50 \pm 6,788$ & 0,553 \\
\hline Cortisol (mcg dl ${ }^{-1}$ ) & $8,036 \pm 0,159^{\mathrm{a}}$ & $8,810 \pm 0,159^{b}$ & $8,807 \pm 0,159^{b}$ & 0,007 \\
\hline
\end{tabular}

Lesões da pele são medidas por um escore de 4 pontos: 1 e 2 representam valores aceitáveis e 3 e 4 inaceitáveis. LD- Longissimus dorsi; SM- Semimembranosus; CRA= capacidade de retenção de água; CPK= creatina fosfoquinase.

O sistema de embarque e desembarque não afetou significativamente a incidência de lesões severas na carcaça, já que não foram constados escores 3 e 4 . Ambos os sistemas apresentaram facilidade no manejo durante o embarque na granja, sem necessidade de intervenções agressivas, estando de acordo com FAUCITANO (1996), que encontrou alta correlação $(\mathrm{P}<0,01)$ entre a incidência de lesões nas carcaças e a qualidade das instalações para auxiliar na condução dos suínos.

Não houve diferença nos escores de lesões nas carcaças $(\mathrm{P}>0,05)$, embora os suínos embarcados e desembarcados utilizando o modelo de veículo com carroceria com piso fixo tenham apresentado valores médios dos escores de lesões nas carcaças superiores, quando comparado ao veículo com carroceria com piso móvel, no entanto, não diferiram $(\mathrm{P}>0,05)$. Os dados observados no presente trabalho não corroboram os resultados obtidos por DALLA COSTA (2006) e DALLA COSTA et al. (2007a), que encontraram efeito do modelo de carroceria sobre o incidência de lesões de pele e na carcaça.

Não houve interação $(\mathrm{P}>0,05)$ entre 0 sistema de embarque e desembarque e linhagens genéticas na incidência de lesões na carcaça. Os resultados obtidos neste experimento para a incidência de lesões estão de acordo com NANI COSTA et al. (1999) que também não verificaram diferença desta variável $(\mathrm{P}>0,05)$ nos grupos de suínos embarcados com plataforma hidráulica e rampa fixa.

Neste experimento, admitiu-se que todos os suínos foram expostos a manejos semelhantes nas granjas e realizados os controles de todos os procedimentos de transporte, descanso e condução para a área de insensibilização, havendo variação somente no sistema de embarque e desembarque. No entanto, pode haver interpretações equivocadas na incidência das lesões provocadas durante a criação na granja e as proporcionadas durante o embarque, transporte e desembarque, já que a incidência de lesões na granja não foi avaliada nos suínos.

Analisando os valores médios de $\mathrm{pH}$, cor, capacidade de retenção de água e perda por exsudação, constata-se que não houve efeito significativo $(\mathrm{P}>0,05)$ do sistema de embarque e desembarque para as linhagens genéticas A, B e C. Para todas as características físico-químicas avaliadas, o sistema de embarque e desembarque não influenciou na qualidade da carne, assim como não houve interação $(\mathrm{P}>0,05)$ entre o sistema de embarque e desembarque e as linhagens genéticas. Resultados semelhantes também foram constatados por NANI COSTA et al. (1999), que 
não encontraram diferenças nas características de qualidade da carne, quando avaliaram o efeito do sistema de embarque e desembarque com plataforma hidráulica e rampa fixa em suínos provenientes de duas granjas na Itália. Os mesmos autores também não constataram diferença nas características do $\mathrm{pH}$ final da carne, diferindo dos resultados obtidos por DALLA COSTA et al. (2007b), que observaram valor médio de $\mathrm{pH}$ final significativamente menor nos suínos que foram embarcados no modelo de veículo com dois andares (carroceria dupla com piso fixo), quando comparado ao modelo de veículo com um único andar (carroceria simples).

Os resultados encontrados neste trabalho demonstram que se os procedimentos de manejo durante o embarque nas granjas e o desembarque no frigorífico forem bons, com distância de transporte curta e o tempo de descanso no frigorífico próximo a 6 horas, não há interferência significativa do sistema de embarque e desembarque na qualidade da carne dos suínos provenientes das linhagens genéticas A, B e C. Em monitoramento realizado por AVEROS et al. (2007), os níveis de estresse dos suínos durante a etapa de embarque, transporte, desembarque e descanso no frigorífico aumentaram significativamente durante o transporte e diminuíram durante o descanso $(\mathrm{P}<0,001)$. Avaliações feitas por BRADSHAW et al. (1996) também comprovaram a diminuição dos níveis de estresse durante o descanso. Ainda de acordo com o mesmo autor, o estresse proporcionado pelo transporte pode ser recuperado após $5 \mathrm{~h}$ de descanso.

O sistema de embarque e desembarque afetou significativamente a concentração do cortisol plasmático, conforme descrito na tabela 1 , porém essa diferença não foi significativa para provocar alterações na atividade enzimática (lactato-desidrogenase e creatina fosquinase) e causar alterações na musculatura. Os suínos que foram embarcados no veículo com carroceria com piso móvel apresentaram níveis mais baixos de cortisol $(\mathrm{P}=0,001)$, quando comparado ao sistema de embarque e desembarque com piso fixo, o que demonstra que os animais submetidos a S1 apresentaram menor nível de estresse psicológico. O aumento nos parâmetros fisiológicos do estresse, como o cortisol plasmático, está diretamente associado com estresse psicológico, e lactato ou creatina fosfoquinase com estresse físico (WARRISS, et al. 1994). AVEROS et al. (2007) também constataram aumento significativo $(\mathrm{P}<0,001)$ na concentração de cortisol, conforme os suínos enfrentaram situações mais estressantes no transporte.

Os valores médios encontrados para cortisol são condizentes com situações do manejo de mínimo estresse. Conforme BROWN et al. (1998), suínos abatidos em condições estressantes trazem resultados de cortisol próximos a 17,02 $\mu \mathrm{g} 100 \mathrm{~mL}^{-1}$ e, em situações de mínimo estresse, $7,62 \mu \mathrm{g} 100 \mathrm{~mL}^{-1}$, semelhante aos valores médios encontrados neste trabalho para S1 e S2 nas linhagens A, B e C.

Os suínos da linhagem genética $A$ apresentaram valores médios de cortisol inferior $(\mathrm{P}<0,05)$, quando comparado às linhagens $\mathrm{B}$ e $\mathrm{C}$. No entanto, esse efeito não pode ser atribuído somente à linhagem genética, já que os suínos se encontravam em granjas diferentes. Apesar de admitirmos que fossem expostos a manejos semelhantes, pode haver efeito da granja na reatividade dos suínos.

Não houve interação $(\mathrm{P}>0,05)$ entre o efeito do sistema de embarque e desembarque e a genética para os parâmetros fisiológicos do estresse. Os valores médios encontrados para lactato e creatina fosfoquinase foram superiores no S1 quando comparado ao S2, no entanto, o sistema de embarque e desembarque não afetou significativamente esses dois parâmetros $(\mathrm{P}>0,05)$. Os valores médios de lactato e creatina fosfoquinase também não diferiram $(\mathrm{P}>0,05)$ entre as três linhagens genéticas. $\mathrm{O}$ estresse gerado pelo sistema de embarque e desembarque nas linhagens genéticas $\mathrm{A}$, B e $\mathrm{C}$ foi insuficiente para causar alterações nos parâmetros de estresse físico (lactato e CPK). Isso corrobora NANI COSTA et al. (1999) que também não constataram em sua pesquisa efeito significativo do sistema de embarque nos parâmetros de estresse físico (lactato e glicogênio muscular) entre os grupos de suínos embarcados com plataforma hidráulica e rampa fixa. No entanto, pode ter havido interferência do tempo de descanso no frigorífico, minimizando o estresse provocado durante o embarque e desembarque, já que lá os animais permaneceram por no mínimo 6 horas, de acordo com as recomendações exigidas pelo Serviço de Inspeção Federal do frigorífico (BRASIL, 1995).

\section{CONCLUSÃO}

Os níveis de estresse causados pelos sistemas de embarque na granja e desembarque no frigorífico foram insuficientes para causar alterações nos indicadores de estresse físico e nas características de qualidade da carne nos suínos das linhagens A, B e C. Ambos os sistemas utilizados geraram baixos níveis de estresse e proporcionaram facilidade de manejo.

\section{AGRADECIMENTOS}

À Fundação de Amparo à Pesquisa do Estado de São Paulo (FAPESP).

Ciência Rural, v.42, n.3, mar, 2012. 


\section{COMITÊ DE ÉTICA E BIOSSEGURANÇA}

Este projeto foi aprovado pela Câmara de Ética em Experimentação Animal (CEUA 38/2004) da Faculdade de Medicina Veterinária e Zootecnia da Universidade Estadual Paulista Júlio de Mesquita Filho, Botucatu, São Paulo.

\section{REFERÊNCIAS}

AVEROS, X. et al. Serum stress parameters in pigs transported to slaughter under commercial conditions in different seasons. Veterinarni Medicina, v.52, n.8, p.333-342, 2007. Obtido via base de dados Agricultural Journals. Acesso em: 08 out. 2011 Online. Disponível em: <http://www.agriculturejournals.cz/ publicFiles/00314.pdf $>$.

BARTON GADE, P.A. et al. Methods of improving welfare and meat quality by reducing stress and discomfort before slaughter - methods of assessing meat quality. In: EUSEMINAR NEW INFORMATION ON WELFARE AND MEAT QUALITY OF PIGS AS RELATED TO HANDLING, TRANSPORT AND LAIRAGE CONDITIONS, 1996 Landbauforschung Volkenrode, Sonderheft, Denmark. Proceedings... Landbauforschung Volkenrode, Sonderheft: EU SEMINAR, 1996. p.23-35.

BENCH, C. et al. The welfare of pigs during transport. In: SCHAEFER, A.; FAUCITANO, L. Welfare of pigs - from birth to slaughter. The Netherlands: Wageningen Academic Publishers, 2008. Cap.06, p.161-187.

BERTOLONI, W. et al. Avaliação de diferentes híbridos suínos submetidos à insensibilização elétrica e gasosa (CO2). Parte 1 mensuração de indicadores sanguíneos de estresse. Ciência e Tecnologia de Alimentos, v.26, n.3, p.564-570, 2006a. Disponível em: <http://dx.doi.org/10.1590/S0101-20612006000300013>. Acesso em: 28 out. 2011. doi: 10.1590/S010120612006000300013.

BERTOLONI, W. et al. Avaliação de diferentes híbridos suínos submetidos à insensibilização elétrica e gasosa (CO ). Parte 2 mensurações objetivas de qualidade. Ciência e Tećnologia de Alimentos, v.26, n.2, p.343-354, 2006b. Disponível em: http:/ /dx.doi.org/10.1590/S0101-20612006000200017>. Acesso em: 24 out. 2011. doi: 10.1590/S0101-20612006000200017.

BRADSHAW, R.H. et al. Stress and travel sickness in pigs: effects of road transport on plasma concentrations of cortisol, beta-endorphin lysine and vasopressin. Journal of Animal Science, v.63, p.507-516, 1996.

BRASIL. Ministério da Agricultura, Pecuária e Abastecimento. Secretaria de Desenvolvimento Rural. Coordenação de Informação Documental Agrícola. Portaria n.711, Normas técnicas de instalações e equipamentos para abate e industrialização de suínos. Brasília, p.1-100, nov. 1995.

BROWN, S.N. et al. Meat quality in pigs subjected to minimal preslaughter stress. Meat Science, v.49, p.257-265, 1998. Disponível em: <http://dx.doi.org/10.1016/S0309-1740(97)00146-0>. Acesso em: 29 ago. 2011. doi: 10.1016/S0309-1740(97)00146-0.

DALLA COSTA, O.A. Efeitos do manejo pré-abate no bem-estar e na qualidade de carne de suínos. 2006. 162p. Tese (Doutorado em Zootecnia) - Faculdade de Ciências Agrárias e Veterinárias, UNESP, Jaboticabal, SP.
DALLA COSTA, O.A. et al. Effects of the season of the year, truck type and location on truck on skin bruises and meat quality in pigs. Livestock Science, v.107, p.29-36, 2007a. Disponível em: <http://dx.doi.org/10.1016/j.livsci.2006.08.015>. Acesso em: 26 set. 2011. doi: 10.1016/j.livsci.2006.08.015.

DALLA COSTA, O.A. et al. Modelo de carroceria e seu impacto sobre o bem-estar e a qualidade da carne dos suínos. Ciência Rural, v.37, n.5, p.1418-1422, 2007b. Disponível em: <http:/ /dx.doi.org/10.1590/S0103-84782007000500031>. Acesso em: 12 set. 2011 . doi: 10.1590/S0103-84782007000500031.

EUROPEAN CONVENTION (EC). Council Regulation n.1/ 2005 (2005): On the protection of animals during transport and related operations and amending Directives 64/432/EEC and 93/119/EC and Regulation (EC) No 1255/97. Official Journal of the European Union, L 3, 22/12/2004, p.00010044 .

FAUCITANO, L. Effects of environment on the animal welfare and the quality of the pig production. 1996 243f. PhD (thesis) - University Bologna, Italy.

GRANDIN, T. The feasibility of using vocalization scoring as an indicator of poor welfare during cattle slaughter. Applied Animal Behaviour Science, v.56, n.2-4, p.121-128, 1998. Disponível em: <http://dx.doi.org/10.1016/S0168-1591(97)00102-0>. Acesso em: 26 out. 2011. doi: 10.1016/S0168-1591(97)00102-0.

HONIKEL, K.O. Reference methods for the assessment of physical characteristics of meat. Meat Science, v.49, p.447457, 1998. Disponível em: <http://dx.doi.org/10.1016/S03091740(98)00034-5>. Acesso em: 18 out. 2011. doi:10.1016/ S0309-1740(98)00034-5.

NANNI COSTA, L. et al. Influence of loading method and stocking density during transport on meat and dry-cured ham quality in pigs with different halothane genotypes. Meat Science, v.51, n.4, p.391-399, 1999. Disponível em: <http:/ /dx.doi.org/10.1016/S0309-1740(98)00160-0>. Acesso em: 18 out. de 2011. doi: 10.1016/S0309-1740(98)00160-0.

ORGANIZAÇÃO INTERNACIONAL DE EPIZOOTIAS (OIE). Terrestrial Animal Health Code - Transport of animals by land. 2011. Cap.7.3, 17p. Acesso em: 19 out. 2011. Online. Disponível em: <http://www.oie.int/fileadmin/Home/ eng/Health_standards/tahc/2010/en_chapitre_1.7.3.pdf >.

SAS INSTITUTE INC. System for Microsoft Windows. Release 9.1. Cary, NC, 2003. (cd-rom).

SHAW, F.D.; TUME, R.K. The assessment of pre-slaughter and slaughter treatments of livestock by measurement of plasma constituents - A review of recent work. Meat Science. v.32, n.3, p.311-329, 1992. Disponível em: <http://dx.doi.org/ 10.1016/0309-1740(92)90095>. Acesso em: 05 out. 2011. doi: 10.1016/0309-1740(92)90095-L.

WARRISS, P.D. et al. Relationships between subjective and objective assessments of stress at slaughter and meat quality in pigs. Meat Science, v.38, n.2, p.329-340, 1994. Disponível em: $<$ http://dx.doi.org/10.1016/0309-1740(94)90121-X>. Acesso em: 05 out. de 2011. doi:10.1016/0309-1740(94)90121-X. 